\title{
STRONGMAR Summer School 2016 - Joining theory with a practical application in Underwater Archeology
}

Mario Monteiro Marques ${ }^{1}$, Augusto Salgado ${ }^{I}$, Victor Lobo ${ }^{I}$, Rodolfo Santos Carapau ${ }^{1}$, Alexandre Valerio Rodrigues ${ }^{1}$ Marc Carreras ${ }^{3}$, Joseta Roca ${ }^{3}$, Narcís Palomeras ${ }^{3}$ Natàlia Hurtós $^{3}$, Carles Candela ${ }^{3}$ Alfredo Martins ${ }^{2,4}$, Aníbal Matos $^{2}$ ,5, Bruno Ferreira ${ }^{2}$, Carlos Almeida ${ }^{2}$, Filipe Aranda de Sa ${ }^{2}$, José Miguel Almeida ${ }^{2,4}$, Eduardo Silva $^{2,4}$.

${ }^{1}$ CINAV, Portuguese Navy Research Center, Almada, Portugal

${ }^{2}$ INESC TEC, Porto, Portugal

${ }^{3}$ Universitat de Girona

${ }^{4}$ ISEP - School of Engineering, Polytechnic Institute of Porto (Porto, Portugal)

${ }^{5}$ FEUP - Faculty of Engineering, University of Porto (Porto, Portugal)

\begin{abstract}
This paper aims at presenting the STRONGMAR Summer School 2016 that took place at the Base Naval de Lisboa, of the Portuguese Navy. The STRONGMAR project ideal motivates the development of maritime and marine science research and technology through the knowledge transfer between INESC TEC and promising, and prestigious, leading research European institutions. This process takes place through theoretical lectures and training, and via experimental application of the concepts discussed in order to further develop technology related to the sea environment. The practical application of the STRONGMAR project ideal takes place during events such as summer schools, winter schools, thematic workshops and scientific conferences. The STRONGMAR Summer School 2016 approaches the subject of "Introduction to Advanced Marine Technologies", providing a strong component of practical applications in underwater archaeology. It develops the study of unmanned systems applied to underwater archaeology, through the use of unmanned underwater vehicles. As a whole, this paper describes the Summer School experience, providing some results and greater insight on the topic of underwater archaeology.
\end{abstract}

Keywords-STRONGMAR Summer School, maritime technology, underwater archaeology.

\section{INTRODUCTION}

Nowadays, technology has been developing towards the use of unmanned systems. The potential behind unmanned systems allows for costs reduction, human risk reduction and an increase of capabilities at performing multiple tasks such as search and rescue, surveillance, scientific exploration, payload delivery, among many other [1] [2], [3].

Regarding the advances in maritime technology, the STRONGMAR project (Strengthening Maritime Technology Research Center), funded by the European Commission under the Horizon 2020 EU Framework Program for Research and
Innovation, aims to "create solid and productive links in the global field of marine science and technology between INESC TEC and established leading research European institutions, helping raising its staff's research profile and its recognition as a European maritime research center of excellence [4]. The STRONGMAR project's development takes place during events like summer schools, winter schools, thematic workshops and scientific conferences, among others, at the following events:

- Summer schools: "Introduction to advanced marine technologies", "Underwater marine perception" and "Deep sea marine perception and operation";

- Winter schools: "Underwater sensing science", "Underwater vision and robotics", "Underwater system and communications science", "Advanced Underwater perception" and "Advanced autonomous marine operations";

- Thematic workshops: "Ocean technology", "Sensing the deep sea", "Underwater distributed perception" and "Robotics for underwater mining - from exploration to exploitation";

- Scientific conferences: "2016 STRONGMAR conference - A sea of science", "2017 STRONGMAR conference - A sea of technology" and "2018 STRONGMAR conference - A sea of innovation";

- Technology transfer workshops with stakeholders

These events serve as essential opportunities to raise the five main pillars of the STRONGMAR concept: Multidisciplinary collaboration network, broad and focused training, thematic workshops, hands-on training, and cross-fertilization. The structure of the STRONGMAR project works towards the achievement of established general, functional and operational objectives. The general objectives are based on knowledge transfer, exchange of good practices, and the provision of services across European academic and industrial communities, and therefore enhancing INESC TEC, and partners, as a valuable maritime research reference. The functional objectives are focused on raising INESC TEC's capabilities related to low cost sensors, endurance and long term navigation, 
communications and acoustics, and structures and manipulators. These objectives rely heavily on the development of knowledge transfer and cross-fertilization between scientific partners, in order to provide more interaction across the scientific community, regarding the field of maritime technology. Besides INESC TEC as the coordinating entity, the STRONGMAR project relies on a consortium composed by: CINTAL (Portugal), the Heriot-Watt University (Scotland, United Kingdom), the NATO Science \& Technology Organization (Belgium), the University of Girona (Spain) and the University of Aberdeen (Scotland, United Kingdom) [5].

The development of unmanned systems related to maritime technology is primarily focused on the development of unmanned surface vehicles (USV) and unmanned underwater vehicles (UUVs). The STRONGMAR Summer School 2016 addressees this technology, in particular the advances on UUV technology. UUVs are robotic assets which have no local human operator onboard and travel underwater. They are typically divided into two separate classes: remotely operated vehicles (ROV) and autonomous underwater vehicles (AUV). The main difference between the two separate classes is the independence that the AUVs have when it comes to navigation, sensing and autonomous capability of making decisions in the performance of their tasks, whereas ROVs are remotely controlled by an operator via cable, fiber optics, radio, or other means [6]. This technology can be employed in tasks such as: oceanographic surveys, bathymetric measurements, underwater maintenance activities, maritime reconnaissance, communication relaying, navigational aids, submarine track and trail, ocean floor mapping, underwater archeology, and other applications [7] [8] [9].

The STRONGMAR Summer School 2016 purpose is aligned with the STRONGMAR project's training strategy since it allows for experts in fields related to marine technologies to share knowledge and experiences related to each one's personal research. This flow of information interchange is key to further development related to the sea environment related challenges and to the growth of the STRONGMAR project. According to this premise, this summer school discusses and develops topics like knowledge-transfer and technology-based entrepreneurship, underwater archeology, visual and acoustic perception, underwater acoustics, underwater sensing, optical sensing and multi-target tracking, and it also allows for training using the SPARUS II AUV [4].

This paper begins with an introduction to the STRONGMAR project and the summer school, followed by section II which describes the STRONGMAR summer school 2016 as a whole. Section III presents a detailed approach of the practical tests that took place, while section IV highlights underwater archaeology in the context of the summer school. Finally, the conclusions section resumes and enhances the importance of the summer school, indicating the main achievements, lessons learned and future work.

\section{SYLLABUS OF THE COURSE}

The summer school 2016 took place at Escola Naval (Navy School), inside Base Naval de Lisboa (Lisbon Navy
Base) which is located in Almada and it is a military base of the Portuguese Navy.

The main focus of the course is the "Introduction to advanced marine technologies", which covers underwater technology and exploration, as well as the study of acoustics and sensing. The course is divided in two main parts, an introduction to advanced marine technologies through a series of lectures, followed by a period dedicated to experiments with an AUV and at the Robotics EXercise 2016 (REX16). REX is an event dedicated to perform various field experiments with multiple unnamed systems in the context of the Portuguese Navy concept of operations [10].

The course aims to develop the scope of the STRONGMAR project, allowing for interaction between specialists, contributing to increase the knowledge at the field of marine technology.

The course had the duration of two weeks. The first week was mainly filled by lectures until the last day of the week, in which began the REX16. The second week presented the experimental part of the course, with the REX16 and with Sea training with AUVs.

Day one of the first week began with two lectures, the first by Prof. Victor Lobo, from Centro de Investigação Naval (CINAV), and the second by Joseta Roca, from the University of Girona (UdG) - Girona Underwater Vision and Robotics (CIRS). Prof. Victor Lobo's lecture was about the "R\&D activities at the Portuguese Navy", which aimed to introduce some of the activities CINAV was performing, and Joseta Roca's lecture was titled "Knowledge transfer: the story of our underwater technology", which served to introduce the UdG's experiences to the INESC TEC, being its AUV lab expertise and prototypes GIRONA 500 AUV and SPARUS II AUV, and being other project management capabilities, in order to transfer knowledge and locate possible collaboration points.

Day two introduced two lectures by Captain Alves Salgado, from CINAV, and by Matias Valdenegro, from the Heriot Watt University. Captain Salgado's lecture introduces the topic of "Underwater Archaeology", with the aim of presenting HistArC (a Research Program about Maritime History and Archeology of the Conflicts) and to relate the student's experiences with the importance of which can have to the HistArC project .While Matias Valdenegro's lecture dealt with "Visual and Acoustic Perception with Deep Neural Networks", with the main objective of presenting Convolutional Neural Networks and its applications in the underwater technology (and AUV) field, as well as their relation with optical imaging and acoustic data.

The third day introduced two lectures by António Silva and Paulo Santos, both from Centro de Investigação Tecnológica do Algarve (CINTAL). António Silva's lecture, entitled of "Generic introduction to underwater acoustics. Underwater acoustic sensors", developed subjects like technologies, tools and applications related to underwater acoustics with the aim of introducing underwater acoustic ocean sensors, and its whole process since the detection of acoustic signals to its integration in electronic systems. Paulo Santos's lecture developed further the subject "Acoustics for 
underwater sensing", since it was focused on signal processing and its applications, techniques and issues related to underwater acoustics.

The last day of lectures counted on three separate lectures by Daniel Clark and Yan Pailhas, both from the Heriot Watt University, and by Prof. John Watson, from the University of Aberdeen. The lecture presented by Daniel Clark introduced "Multi-target tracking algorithms and applications" which aims to explain the subject and benefits of multi-object filters, and its statistical methodology, as well as its applications. It also develops on to the challenges and solutions related to multisensor fusion applied to space situational awareness. Yan Pailhas lecture develops the "Underwater Acoustics: understanding the environment to increase autonomy" theme, by aiming to explain the relation between sensor design, signal/image processing and the underwater environment, in order to better understand this environment and its hidden challenges. Last but not the least, Prof. John Watson presents "An introduction to Optical Sensing and Measurement Techniques and their application in the offshore environment", a lecture that aims to explain some of the applications of optical sensing in underwater environments, as well as its benefits.

The following days of the course were based upon the experiments by the attending students. It also counted with the participation of speakers Marc Carreras, Narcís Palomeras, Tali Hurtós and Carles Candela, from UdG - CIRS. The experiments took place during REX16 and during a "Sea hands-on training", that involved the use of AUVs MARES and SPARUS II, and a ROAZ II USV.

The course counted with a total of thirteen speakers and trainers from the Portuguese Navy CINAV, CINTAL, Heriot Watt University, UdG and the University of Aberdeen. It also counted with twenty-three students from INESC TEC.

\section{PRACTICAL TESTS}

The practical tests took place during the first and second week of the course, and were integrated into REX16 and the SPARUS II AUV: "Sea hands-on training". REX is an exercise organized by CINAV and the Portuguese Navy, currently at Base Naval de Lisboa, which contributes development of CINAV's scope as well as to develop and create business and investigation ties with other organizations/partners. The focus of REX16 is to explore and develop the potential and benefits of unmanned systems through a number of field experiences, integrated on the Portuguese Navy concept of operations. It employs the ROAZ II Autonomous Surface Vehicle (ASV) and the Modular Autonomous Robot for Environment Sampling (MARES) AUV to test acoustic navigation, maritime wireless communication protocols, autonomy, assessment and environment characterization missions.

An AUV named MARES and an ASV named ROAZ II, both developed at INESC TEC, were deployed to perform environment characterization and assessment missions as well as to test acoustic navigation and test a new maritime wireless communication protocol.

MARES AUV has been of current use since 2007, and it's commonly deployed for environment monitoring, underwater inspection and mapping and surveillance missions, being able to collect georeferenced data through an acoustic positioning system. It has a versatile structure which allows for the transportation of a variety sensors.

ROAZ II ASV is a versatile and adaptable underwater vehicle with multiple manned and unmanned operation modes. It is suited to perform aquatic environment monitoring, bathymetry, data collection and oceanography, security and search and rescue missions. It packs a variety of sensors which enable for precise and autonomous navigation with collision detection, and for tasks such as: environmental modeling, target tracking, automated intrusion detection, identification, area patrol, communications relay and surface support for UUVs. Its reliability is proven throughout the multiple operational missions it has taken part of [10].

In another set of experiments, an AUV developed at the University of Girona and named SPARUS II (Figure 1a), together with ROAZ II ASV, were used not only to perform environment characterization and assessment but also sea bottom mapping and imaging tasks, along other assessments. Both vehicles worked together since the ROAZ provided localization updates to the SPARUS II AUV (Figure 1b).
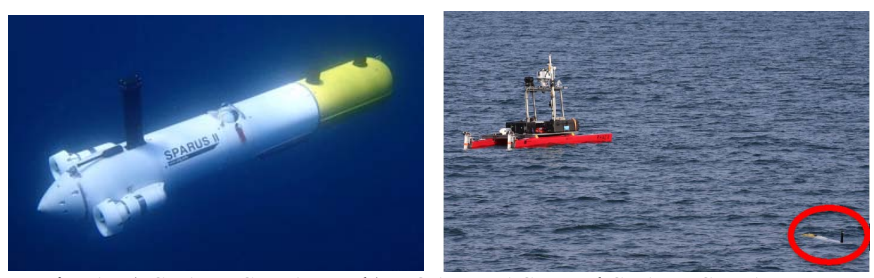

Fig. 1- a) SPARUS II AUV. b) ROAZ II ASV and SPARUS II AUV at surface.

The SPARUS AUV was created by the Underwater Robotics Research Centre (CIRS) at the University of Girona (UdG) in 2010, followed by version II in 2013. It has participated successfully in international competitions and research projects, which has contributed for its commercial availability. SPARUS II AUV [11] is a lightweight hovering vehicle with mission-specific payload area and efficient hydrodynamics for long autonomy in shallow water (200 meters). It combines torpedo-shape performance with hovering capability. It is easy to deploy and to operate. The payload area can be customized by the end-user and it uses an open software architecture, based on ROS [12], for mission programming. Its flexibility, easy operation and openness makes the SPARUS II AUV a multipurpose platform that can adapt to industrial, scientific and academic applications. The key points of the vehicle are: a) torpedo-shape movement with efficient hydrodynamics and long autonomy; b) hovering capability for high maneuverability; c) lightweight vehicle, similar weight and size than underwater gliders; d) easy operation, which can be operated by 2 persons from any small boat; e) mission specific payload: open hardware for equipment integration; f) software architecture based on ROS: open software, available for download, called COLA2 [13]. 
During these experiments, the students and researchers from INESC TEC and the University of Girona were on board a catamaran (Figure 2) with the goal of being trained with the practical operation of the vehicles. The practical experiments during the "Sea hands-on training" were scheduled into to three separate sessions, on board a catamaran where the students and UdG researchers were working. Each session was conducted in a different day and location, as showed in Figure 3.
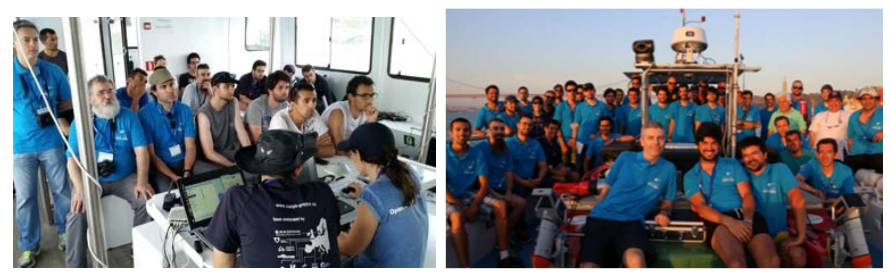

Fig. 2- Hands-on training on board of the catamaran.

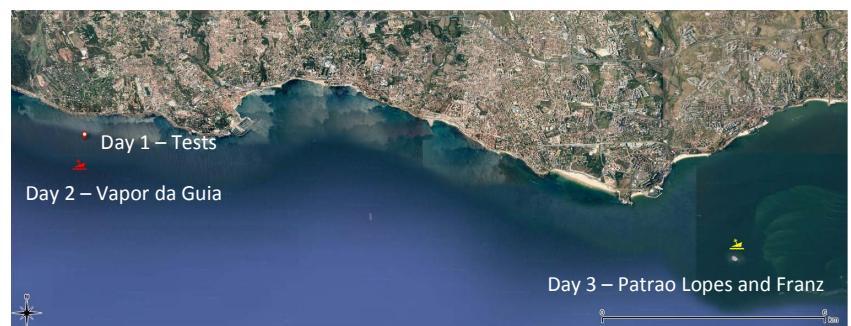

Fig. 3 - Locations where practical exercises were conducted, in the vicinity of the Lisboa shore.

In order to achieve success, there was the need to fulfill objectives such as: defining autonomous missions using a Geographic Information System (GIS) over areas of interest (Figure 4 and 6) resulting in a sea bottom mapping and imaging; configuring and understanding AUV parameters and sensors for the current mission, associated to the AUV deployment and recovery; AUV mission monitoring through acoustic communication; collected data analysis to perform underwater mapping in order to create bathymetry maps (Figure 5 and 7) and sidescan sonar images (Figure 8) using UdG technology.

The experiments conducted during the first day had the goal of demonstrating the operation of Sparus II AUV, from its deployment, to the mission definition and the final representation of results with the graphical user interface. On the second day, experiments were conducted in the site of Vapor da Guia shipwreck. Several trajectories were planned to locate the small relief of the shipwreck. Figures 4 and 5 show one of the executed trajectories and its bathymetry, where the rests of Vapor da Guia can be observed.

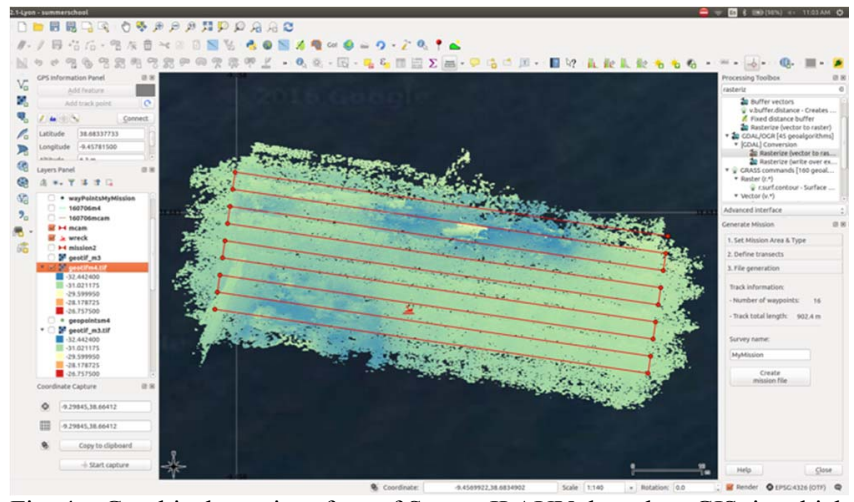

Fig. 4 - Graphical user interface of Sparus II AUV, based on GIS, in which a planned trajectory can be observed on top of the obtained bathymetry.

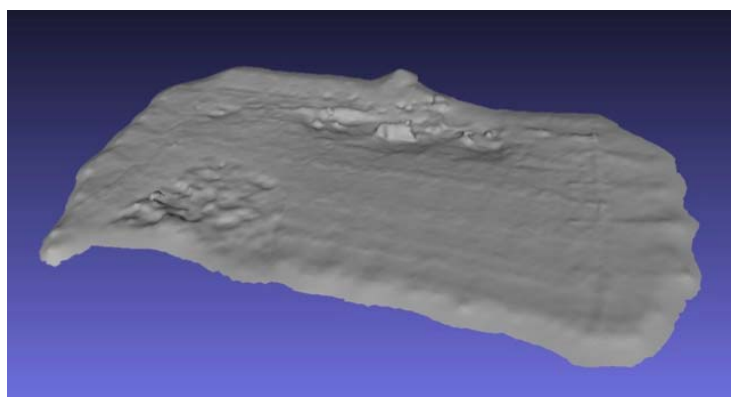

Fig. 5 - Bathymetric map of Vapor da Guia.

During the last day, experiments were conducted in the vicinity of Patrao Lopes and Franz shipwrecks. Several trajectories where defined and executed, as can be seen in Figure 6. Once the data was obtained, it can be observed the result of the bathymetry (Figure 7) and the image generated by the sidescan sonar (Figure 8).

Experiments were successful and the goal of training the students with practical implementations was very constructive

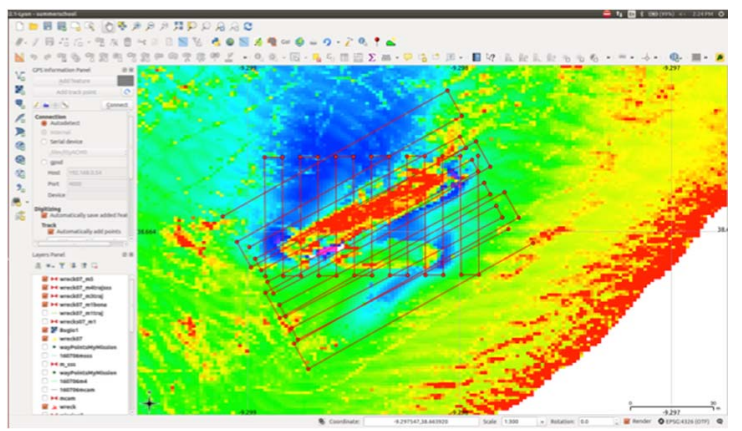

Fig. 6-AUV mission definition on a previous bathymetry using the GIS interface. Corresponds to Patrao Lopes and Franz shipwrecks. 


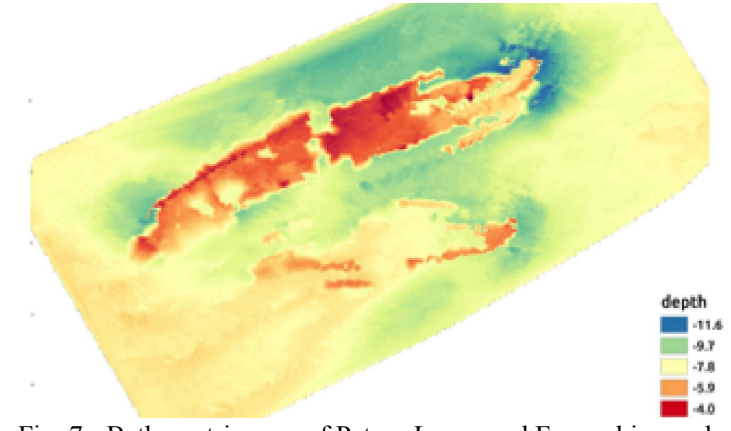

Fig. 7 - Bathymetric map of Patrao Lopes and Franz shipwrecks

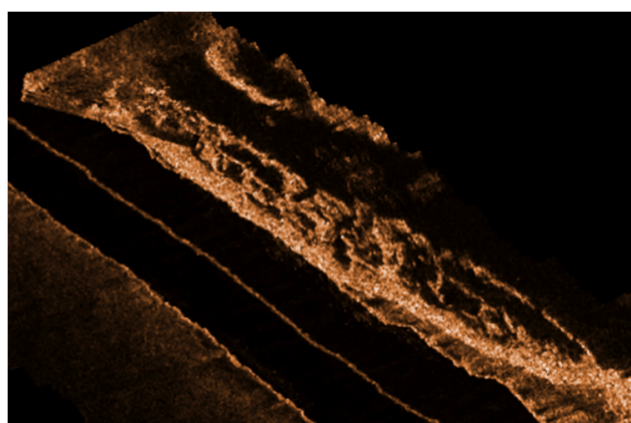

Fig. 8 - Sidescan sonar image of Patrao Lopes and Franz shipwrecks.

\section{APPLICATION ON UNDERWATER ARCHAEOLOGY}

Nautical and underwater archaeology is a branch of archaeology resorting to techniques of access to the underwater environment. Archaeology and underwater heritage seen, in turn, as a scientific practice and a cultural resource, has been the subject of growing concern all over the world. An intervention management is an answer to the scientific and cultural challenges posed by the protection, study and enhancement of the archaeological heritage.

In this environment, different techniques are needed in order to overcome the challenges this difficult environment brings. It is a process that needs more time and is more costly than the archaeology done on dry land. Thus, it is necessary to have efficient techniques, something that diving sometimes does not have. So, in order to overcame the depth, currents, low visibility and other conditions issues, unmanned underwater vehicles can be the solution. These vehicles have tools, ranging from precise navigation to high definition cameras that can give the archaeologist the data as he needs it.

All these underwater archaeology issues were looked at the REX16 series, with tests and demonstrations being made using unmanned underwater vehicles. These activities were mainly conducted near the Bugio lighthouse, at the entrance of the River Tagus, Lisbon, in real shipwrecks. The ex-Portuguese Navy Patrão Lopes that sank in 1936, was the target chosen. The Patrão Lopes was located in 2015, in a biology diving activity, that fortunately also included an underwater archaeologist. Other demonstrations were also made near Guia area, in Cascais. These tests validated the usefulness of the unmanned underwater vehicles in this type of operations, bringing efficiency and other values that could not be achieved with divers.

\section{CONCLUSIONS}

This work served to describe the events that took place during the STRONGMAR Summer School 2016, presenting its motivation and background; lectures and exercises, as well as speakers and students that attended; objectives and results that were established and achieve during the summer school; a description of improvements and impact that the discussed themes and conducted tests had on the applications of underwater archaeology.

It provided a valuable opportunity to connect the theoretical background to the practical experience, and to perform some of the tests during REX16. The SPARUS AUV, MARES AUV and ROAZ II ASV provided promising results, which serve as validation of the potential provided by the use of unmanned systems. This potential was further demonstrated when associating unmanned systems to underwater architecture, proving to be a valuable resource to the future development of archaeological technology.

The Summer School presents an opportunity to the main companies, universities and project developers to provide their knowledge, and to trade experiences and opinions with the goal of cooperating with each other for the sake of technological development.

\section{REFERENCES}

[1] C. Thong, T. Howe and L. Jerome, "Unmanned Technology - The Holy Grail for Militaries?," Pointeer, Journal of the Singapore Armed Forces, 2013.

[2] R. Mendonca, Mario Monteiro Marques, F. Marques, A. Lourenço, P. Rodrigues, P. Santana, V. Lobo, F. Coito, and J. Barata, 'A Cooperative Multi-Robot Team for the Search and Rescue of Shipwrecked Victims', in MTS/IEEE OCEANS 2015, Washington, 2015, pp. 1-6.

[3] R. Mendonca, Mario Monteiro Marques, F. Marques, A. Lourenço, E. Pinto, P. Santana, F. Coito, V. Lobo and J. Barata, 'A Cooperative Multi-Robot Team for the Surveillance of Shipwreck Survivors at Sea', in MTS/IEEE OCEANS 2016, Monterey, 2016, pp. 1-7.

[4] STRONGMAR, "Introduction to Advanced Marine Technologies," STRONGMAR (H2020-TWINN-2015), Almada, 2016.

[5] E. Silva, A. Martins, A. Dias, A. Matos, A. Olivier, C. Pinho, F. Aranda de Sá, H. Ferreira, H. Silva, J. c. Alves, J. M. Almeida, L. Pessoa, M. Ricardo, N. Cruz, N. Dias, P. Mónica, and R. Campos, "Strengthening Marine and Maritime Research and Technology - The STRONGMAR Project," Monterey, USA, 2016.

[6] A. Inzartsev, "Underwater Vehicles," In-Tech Publication, Rijeka, 2009.

[7] R. e. Button, A Survey of Missions for Unmanned Undersea Vehicles, RAND - National Defense Research Institute, 2009. 
[8] O. Yildiz, A. Yilmaz and B. Gokalp, "State-of-the-Art System Solutions for Unmanned Underwater Vehicles," Radioengineering vol.18 nº, Ankara, 2009.

[9] G. C. Rosa, Mario Monteiro Marques, and V. Lobo, 'Unmanned Aerial Vehicles in the Navy: Its Benefits', in $2^{\text {nd }}$ International Conference Sea-Conf 2016, Constanta, 2016, pp. 1-4.

[10] Mario Monteiro Marques, A. Martins, A. Matos, N. Cruz, J. M. Almeida, J. C. Alves, V. Lobo and E. Silva, 'REX 2014 - Robotic Exercises 2014 Multi-robot field trials', in MTS/IEEE OCEANS 2015, Washington, 2015.

[11] M. Carreras, C. Candela, D. Ribas, A. Mallios, L. Magí, E. Vidal, N. Palomeras, and P. Ridao, "SPARUS II, design of a lightweight hovering AUV," in International Workshop on Marine Technology, 2013.

[12] M. Quigley, B. Gerkey, K. Conley, J. Faust, T. Foote, J. Leibs, E. Berger, R. Wheeler, and A. Ng, "Ros: an opensource robot operating system," in Proc. of the IEEE Intl. Conf. On Robotics and Automation (ICRA) Workshop on Open Source Robotics, Kobe, Japan, May 2009.

[13] N. Palomeras, A. El-Fakdi, M. Carreras, and P. Ridao, "COLA2: A Control Architecture for AUVs," IEEE Journal of Oceanic Engineering, vol. 37, no. 4, pp. 695716 , oct 2012. 